\title{
Research Paper \\ The Effects of Co-Administration of Memantine And Vitamin D on Spatial Learning and Memory Impairment in Adult Male Rats Model of Alzheimer's Disease
}

\author{
*Nastaran Zamani ${ }^{1}$, Ahmad Ali Moazedi2, 3 (i) \\ 1. Department of Biology, Faculty of Science, Payame noor university, Tehran, Iran. \\ 2. Department of Biology, Faculty of Science, Shahid Chamran University of Ahvaz, Ahvaz, Iran \\ 3. Stem Cells Technology Research Center, Shahid Chamran University of Ahvaz, Ahvaz, Iran.
}

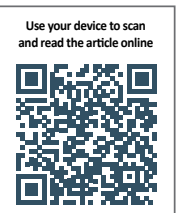

Citation: Zamani N, Moazedi AA. [The Effects of Co-Administration of Memantine And Vitamin D on Spatial Learning and Memory Impairment in Adult Male Rats Model of Alzheimer's Disease (Persian)]. Journal of Arak University of Medical Sciences (JAMS). 2020; 22(6):192-203. https://doi.org/10.32598/JAMS.22.6.5166.2

\section{(i) (8)}

Article Info:

Received: 08 Sep 2019

Accepted: 25 Nov 2019

Available Online: 01 Feb 2020
Key words: Vitamin D, Memantine, Spatial learning, Nucleus basalis of magnocellularis, Alzheimer's disease

\section{A B S T RACT}

Background and Aim Alzheimer's disease is the most common causes of dementia among the elderly people. The aim of this study was to evaluate the synergistic effects of memantine and vitamin D on spatial learning and memory impairment in adult male rat model of Alzheimer's disease.

Methods \& Materials In this experimental study, male Wistar rats were randomly divided into nine groups ( $n=7)$ : $1=$ Control, $2=$ NBM lesion (received bilateral electric lesion of NBM), 3= Sham (the electrode was entered into the NBM with no electric lesion), 4= NBM lesion+ Vehicle Memantine (received saline), 5= NBM lesion+ Vehicle Vitamin D (received saline), $6=$ NBM lesion+ Vehicle Memantine+ Vehicle Vitamin $D$ (received saline plus sesame oil), 7= NBM lesion+Vitamin D; 8= NBM lesion+Memantine, and 9= NBM lesion+Vitamin D+Memantine. After one week, the rats were trained to perform the Y-maze task for five days. Twenty five days after training, a retention test was performed to evaluate their long-term memory. Ethical Considerations This study with research ethics code of "EE/ 97, 24, 3061243/scu.ac.ir" was approved by the Research Ethics Committee of Shahid Chamran University of Ahvaz In Iran.

Results Bilateral NBM lesion reduced spatial learning in comparison with control and sham groups. No effect on spatial learning was observed in NBM lesion+ Vehicle Memantine and NBM lesion+ Vehicle Vitamin D groups compared to the NBM lesion group. Spatial learning and memory in NBM lesion +Vitamin $D+M e m a n t i n e ~ g r o u p ~(P<0.001)$ was significantly improved compared to NBM lesion+Vitamin $D(P<0.01)$ and NBM lesion+Memantine $(P<0.05)$ groups. Moreover, no significant difference was observed between the results in the 5 th day of training and the memory retention at the $30^{\text {th }}$ day.

Conclusion Co-administration of memantine and vitamin D is more effective than memantine or vitamin $\mathrm{D}$ alone in spatial learning and memory improvement in rat model of Alzheimer's disease.

\section{Extended Abstract}

\section{Introduction}

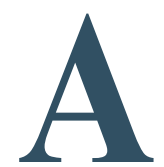

lzheimer's Disease (AD) is the most common cause of dementia among the elderly. Acetylcholine and cholinergic signaling are essential for cognitive functions, including learning and memory. Disorders in cholinergic neurons or postsynaptic acetylcholine receptors have been found to be directly associated with cognitive impairment caused by Alzheimer's disease [2]. Dysfunction or lack of cholinergic cell groups in the basal forebrain, including the Nucleus Basalis Magnocellularis (NBM) in rodents or its equivalent in humans, the Nucleus Basalis of Meynert (NBM), are among the first pathological events in the pathogenesis of

\section{* Corresponding Author}

Nastaran Zamani, PhD.

Address: Department of Biology, Faculty of Science, Payame Noor University, Tehran, Iran

Tel: +98 (916) 3682051

E-mail: na_zamani2000@yahoo.com 
Alzheimer's disease [3]. The present study aims to evaluate the synergistic effects of memantine and vitamin $\mathrm{D}$ on improving spatial learning and memory impairments in adult male rats of Alzheimer's disease model through the bilateral electrical lesion of NBM.

\section{Methods and Materials}

In this experimental study, male Wistar rats were randomly divided into 9 groups ( 7 rats in each): Control group (received no injection or surgery), NBM lesion group (received electrically-induced lesion in NBM), Sham group (the electrode was impaled into the NBM with no lesion), NBM lesion+memantine (saline), NBM lesion+vitamin D (sesame oil), NBM lesion+memantine+vitamin D (saline+sesame oil), and NBM lesion+Vitamin D.

One week after NBM lesion: the last group received 5 $\mu \mathrm{g} / \mathrm{kg}$ vitamin D for 10 days, starting 3 days before NBM lesion, by intraperitoneal injection (IP injection) [13]; the group of NBM lesion + memantine received $3 \mathrm{mg} /$ $\mathrm{kg}$ memantine for 5 days, half an hour before training, by intraperitoneal injection (IP injection) [14]; the group of NBM lesion+memantine+vitamin D received $5 \mu \mathrm{g} / \mathrm{kg}$ vitamin $\mathrm{D}$ for 10 days, starting 3 days before lesion, and $3 \mathrm{mg} / \mathrm{kg}$ memantine for 5 days, half an hour before training, by intraperitoneal injection (IP injection). One week later, rats were trained for 5 days with a Y-shaped maze. Twenty-five days after training, a memory recall test was performed to assess long-term memory.

\section{Results}

Comparison of different groups in the 5 consecutive days of training with One-way Analysis of Variance (ANOVA) showed no significant difference between control and Sham groups in spatial learning in any of the days, whereas bilateral lesion of NBM led to decreased spatial learning $(\mathrm{P}<0.001)$ compared to control and Sham groups. In this study, no change was observed in the spatial learning in vehicle memantine and vitamin D groups compared with the NBM lesion group. On the other hand, the findings of this study showed an improvement in spatial learning and memory of NBM lesion+memantine+vitamin D group $(\mathrm{P}<0.001)$ as compared with the two groups of NBM lesion+vitamin $\mathrm{D}(\mathrm{P}<0.01)$ and $\mathrm{NBM}$ lesion+memantine $(\mathrm{P}<0.05)$. Besides, no significant difference was observed between the results of the fifth day of training and the $30^{\text {th }}$ day memory recall test in either group.

\section{Discussion}

In this study, the bilateral electrical lesion of the Nucleus basalis Magnocellularis (NBM) was used as a model to induce Alzheimer's disease, besides spatial learning and memory impairments in rats. The findings of this study indicated that the combined treatment of vitamin D and memantine through the Intraperitoneal (IP) injection of vitamin D for 10 days (starting 3 days before NBM lesion) and induction of Alzheimer's disease model and intraperitoneal injection of memantine for 5 days (starting 7 days after NBM lesion) had a greater effect on the improvement of spatial learning and memory impairments in the Alzheimer's disease model rats as compared to the administration of these two treatments each alone. Similarly, Di et al. 's studies have shown that $17 \beta$-estradiol, which has neuroprotective effects as similar to vitamin D, leads to decreased neuronal degeneration and increased neuronal survival when combined with memantine treatment in traumatic brain injury model [21].

On the other hand, Ihalainen's studies on rats with Fimbria-fornix lesion have shown a modest increase in acetylcholine levels in neocortex and hippocampus under chronic treatment with memantine and a significant increase under acute treatment with memantine [5]. Therefore, it seems that in the combined treatment of memantine and vitamin D, vitamin D improves memory and learning in Alzheimer's model rats by increasing neuronal protection and inhibiting axonal degeneration by increasing acetylcholine levels.

\section{Ethical Considerations}

\section{Compliance with ethical guidelines}

This study with Ethics Code EE/ 97, 24, 3061243/ scu. ac.ir was approved by the Research Ethics Committee of Shahid Chamran University of Ahvaz.

\section{Funding}

This study received financial support of Shahid Chamran University of Ahvaz.

\section{Authors' contributions}

All authors met standard writing criteria according to the recommendations of the International Committee of Medical Journal Editors (ICMJE). 


\section{Conflicts of interest}

The authors declare that there is no conflict of interest in the present study.

Acknowledgements

The authors would like to thank the Deputy for Research of the Shahid Chamran University of Ahvaz for their support. 


\title{
اثرات تجويز توأم ممانتين و ويتامين D بر اختلالات يادكيرى و حافظه فضايى در مدل بيمارى آلزايمر موشهاى صحوريز توائ مدايتين وبالغ
}

\author{
"نسترن زمانى '(1)، احمدعلى معاضدى ") \\ 1. كروه زيست شئاسى، دانشكده علوم، دانشكاه يهيام نور، تهران، ايران.

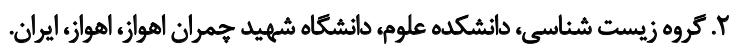

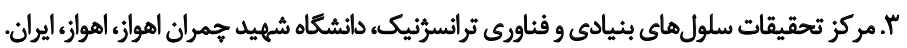

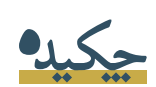

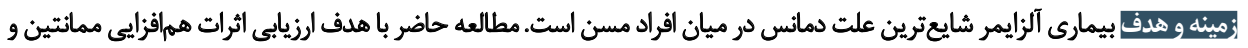

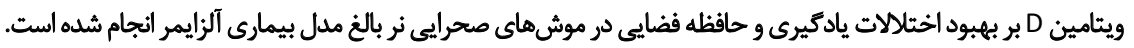

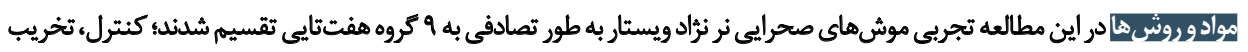

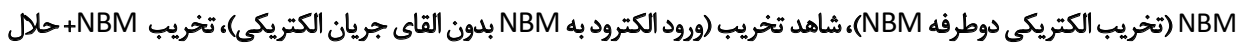

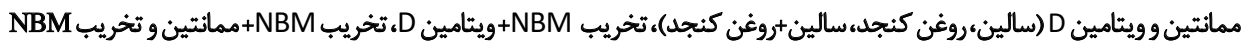

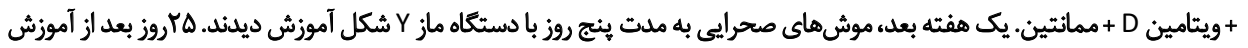

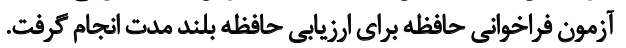

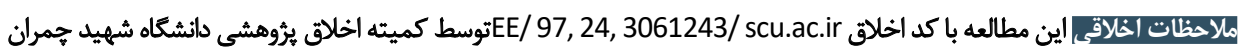

$$
\text { اهواز به تصويب رسيده است. }
$$

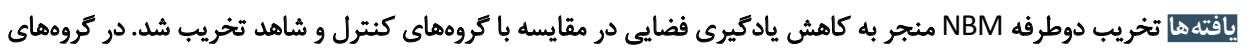

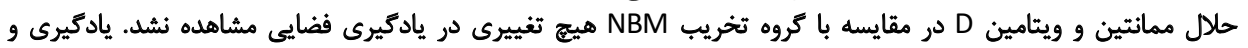

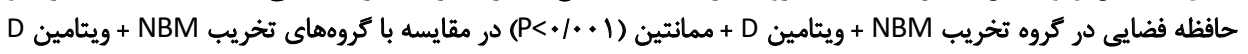

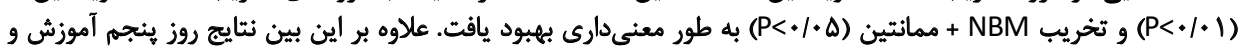

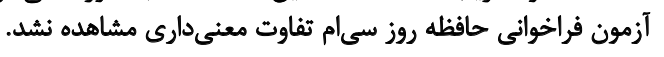

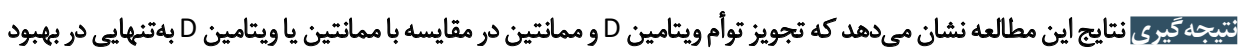

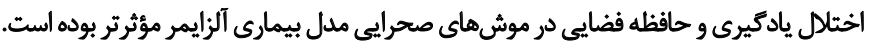

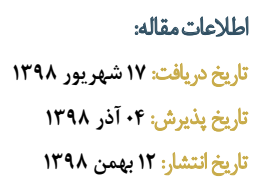

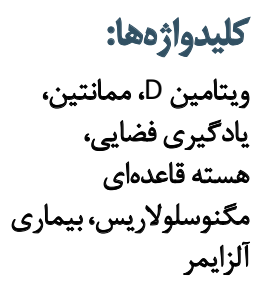

بيمارى آلزايمر در ارتباط هستند.نشان داده شده است كه استيل

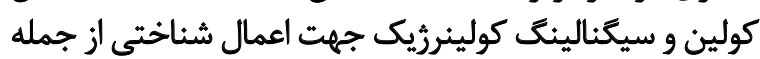

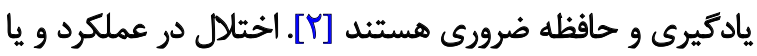

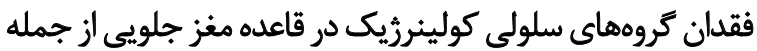

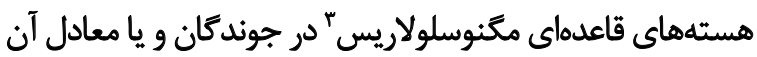

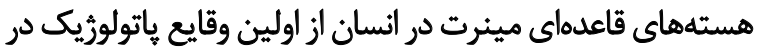

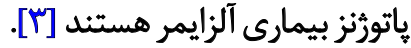
ممانتين آنتاكونيست غيررقابتى، كيرنده NMDA است كه

بيمارى آلزايمر' شايعترين شكل دمانس است كه به واسطه

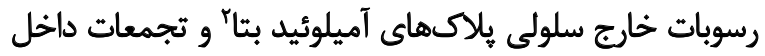

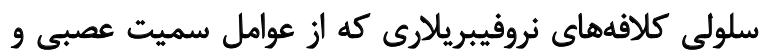

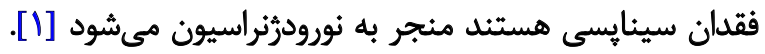

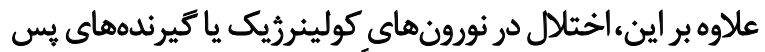

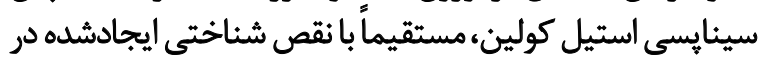

1. Alzheimer's Disease (AD)

2. Beta-Amyloid $(A \beta)$ 
ترتيب توسط حلال سالين 9 • درصد و روغن كنجد به صورت

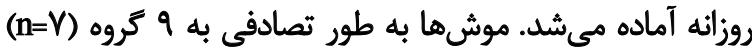

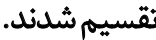

كروه كنترل: كروهى كه تحت هيجگونه تزريق يا جراحى قرار

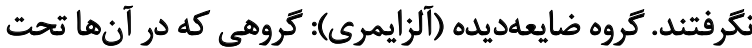

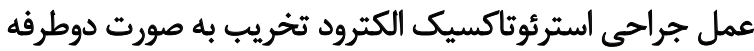

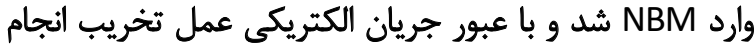

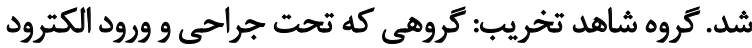

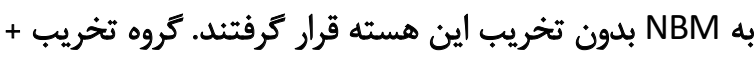

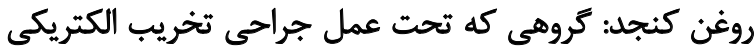

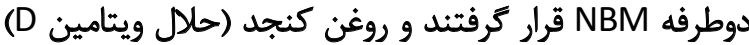

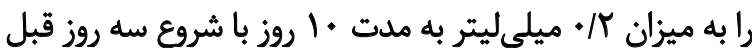

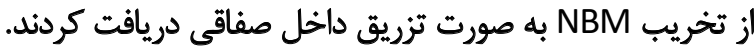

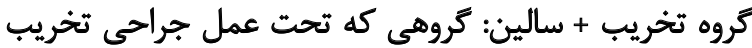

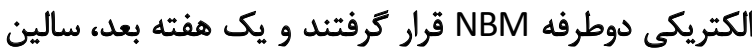

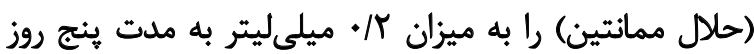

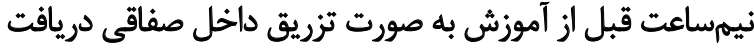

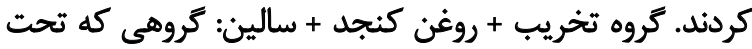

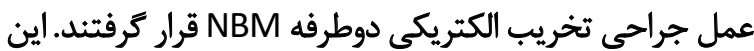

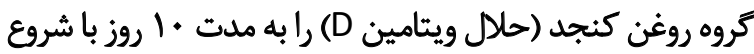

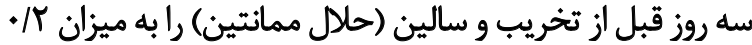

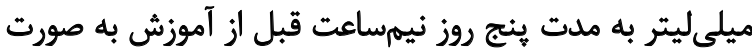
تزريق داخل صفاقى دريافت كردند.

كروه تخريب+ويتامين D: اين كروه تحت عمل جراحي تخريب

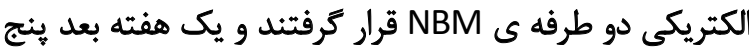

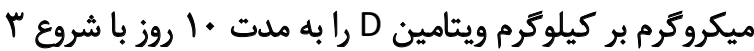

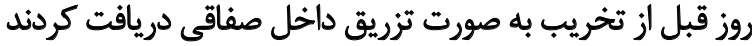

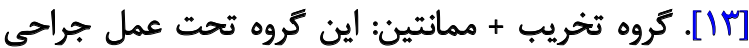

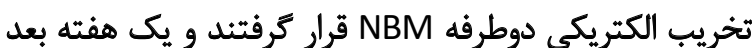

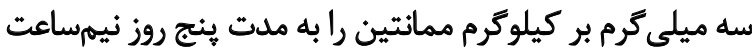

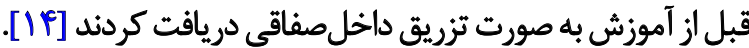

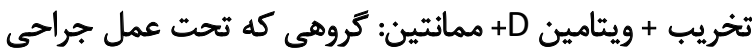

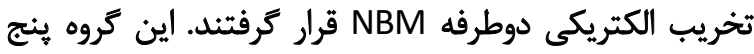

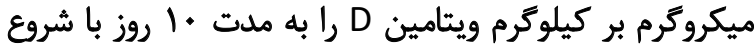

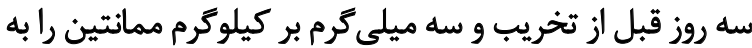

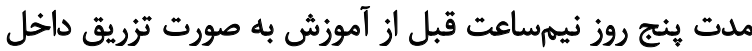

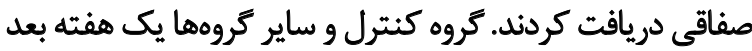

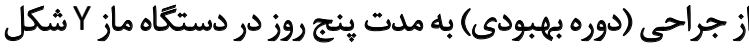

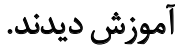

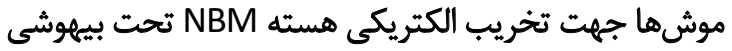

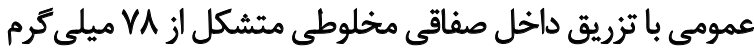

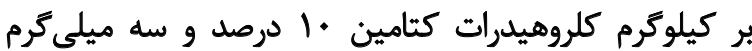

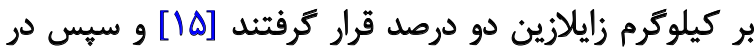

به صورت بالينى جهت درمان بيمارى آلزايمر مورد استفاده قرار

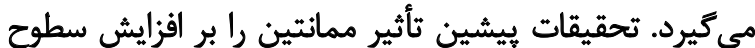

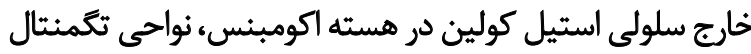

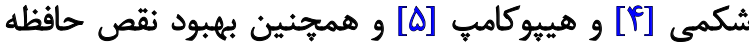

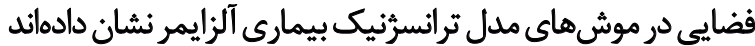

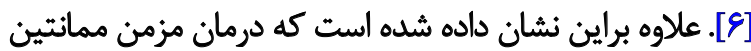

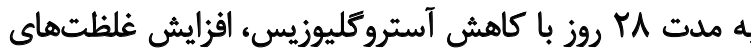
و و GDNF و كاهش آتروفى مغزى ثانويه مى مشود [VDDF].

برخى شواهد نقش مواد غذايى را در بيشكيرى و يا كاهش

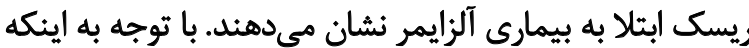

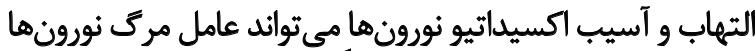

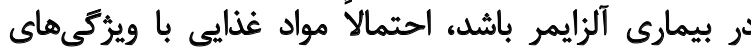

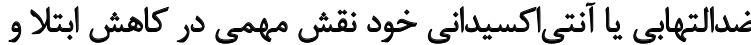

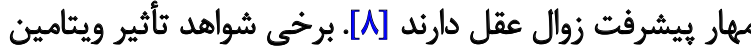

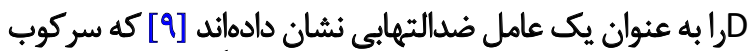

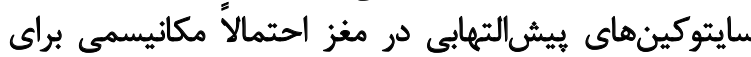

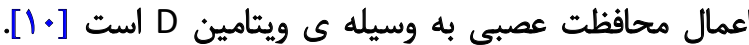

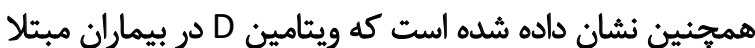

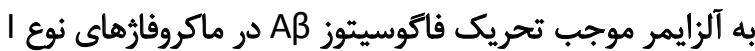

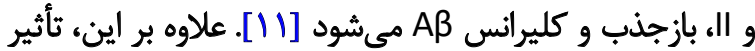

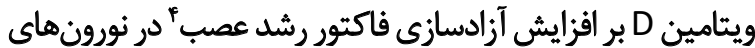

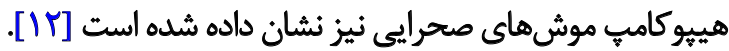

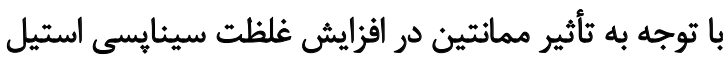

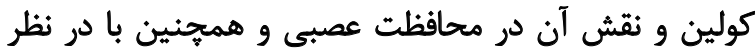

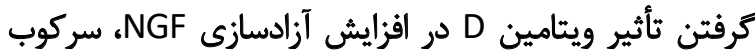

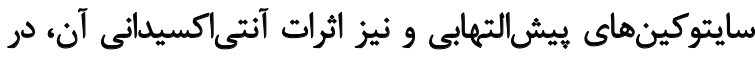

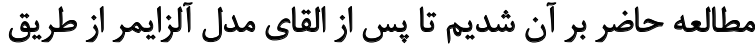

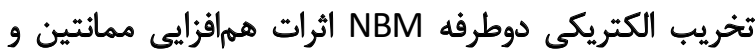

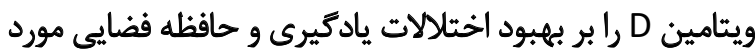

$$
\text { مورسى قرار دهيه. }
$$

در اين مطالعه تجربى از موشهاي صحرايى نر بالغ از نروزاد

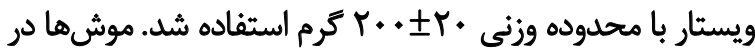

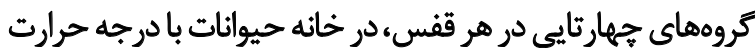

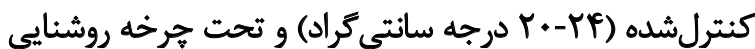

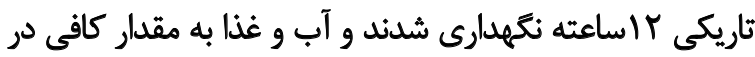

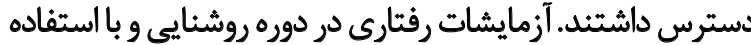

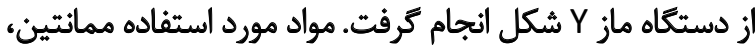

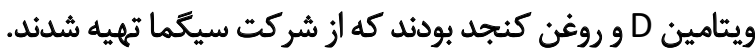

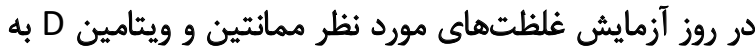

4. Nerve Growth Factor (NGF) 
انتخاب صحيح محسوب مي شود، در غير اين صورت بعد از ينج

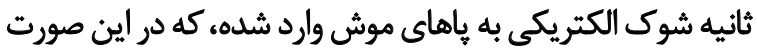

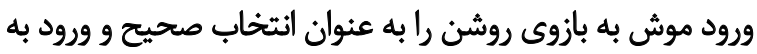

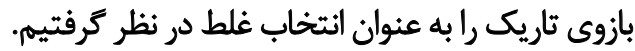

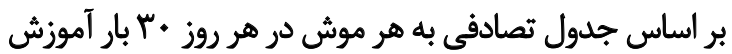

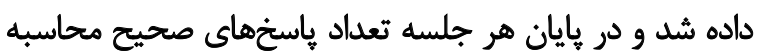

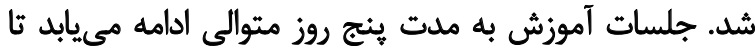

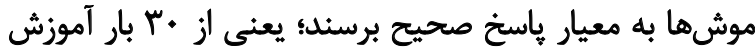

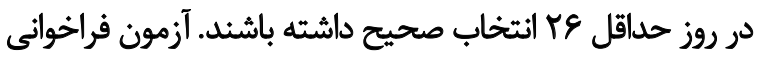

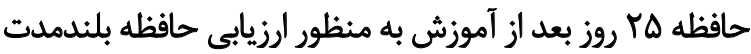

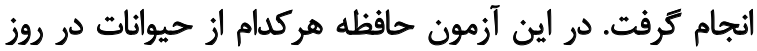

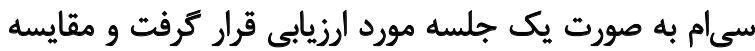

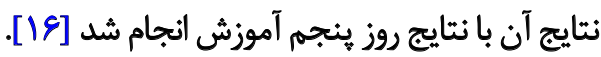

نتايج حاصل از اين يُؤوش به صورت توصيفى و تحليلى با

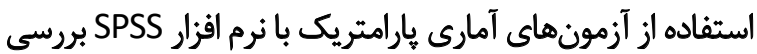

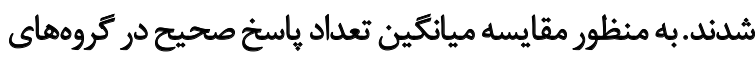

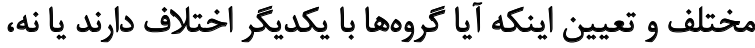

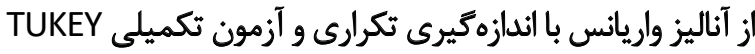

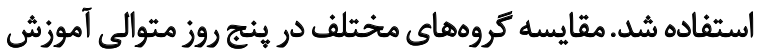

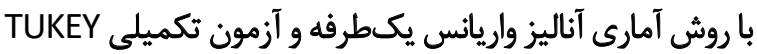

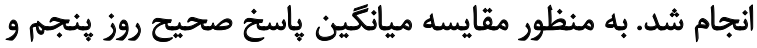

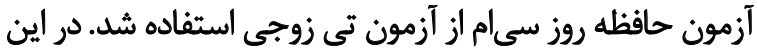

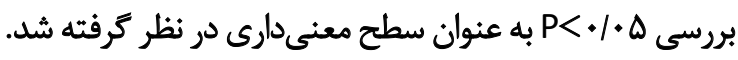

يافتهها

در اين مطالعه نتايج حاصل از آناليز واريانس با اندازمكيرى

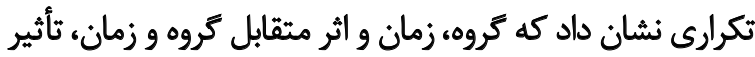

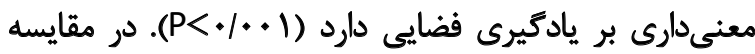

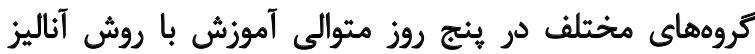

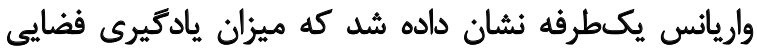

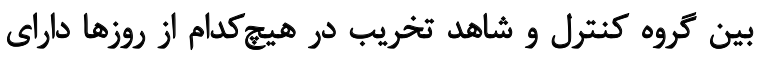

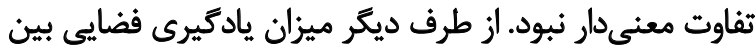

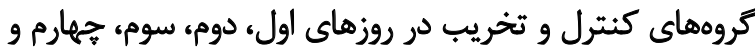

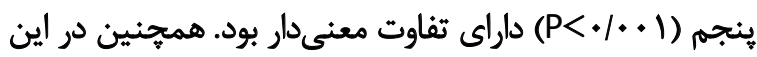

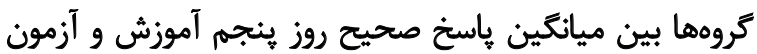

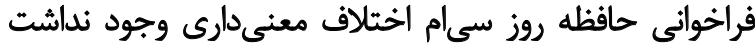

(تصوير شماره ()).

در بررسي اثر حلال ويتامين D (روغن كنجد)، حلال ممانتين

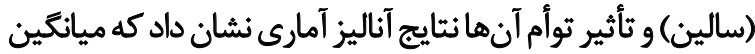

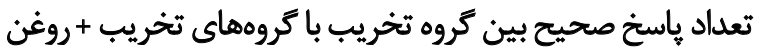

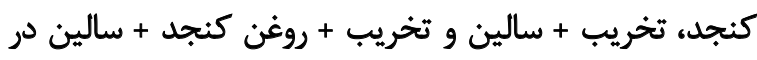

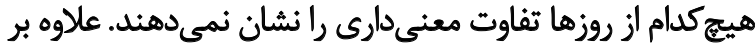
اين، بين ميانكين تعداد ياسخ صحيح روز ينجم آموزش و آزمون
دستكاه استرئوتاكسى (Stoelting, USA) ثابث شدند. ميله

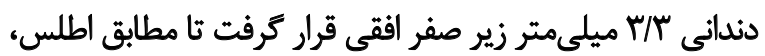

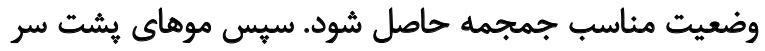

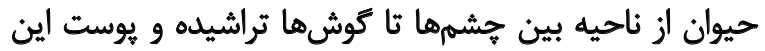

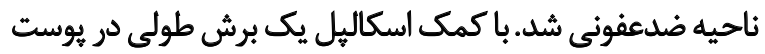

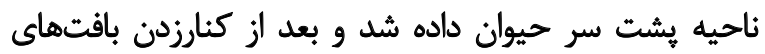

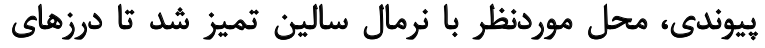

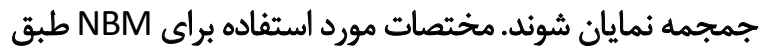

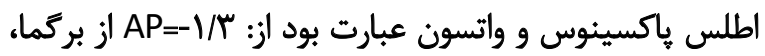
از خط وسط و DL=士Y/A

با استفاده از دستكاه ايجادكننده ضايعه (Lesion Making

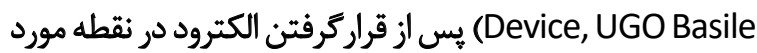

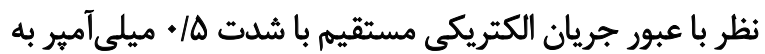

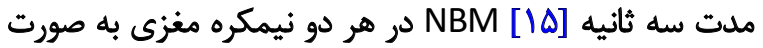
الكتريكى تخريب شد و مدل آلزايمر ايجاد شد ديد.

جهت بررسى روند ياد ميرى و حافظه در مدل هاي آزمايشعاهي

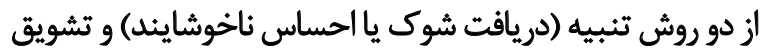

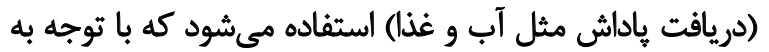

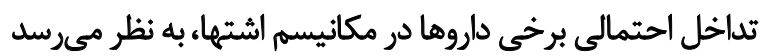

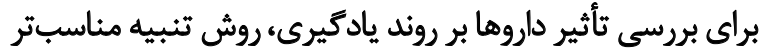

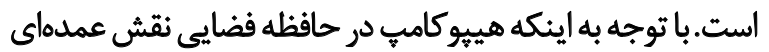

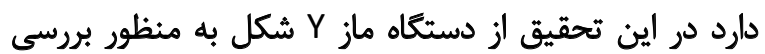

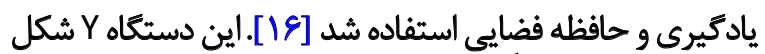

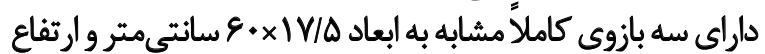

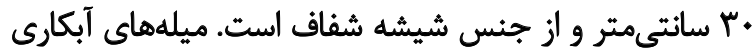

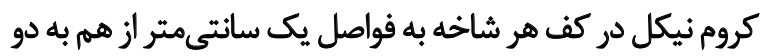
طرف ديوار متصل شده است.

احساس ناخوشايند (شوك الكتريكى) از طريق اين ميلهها

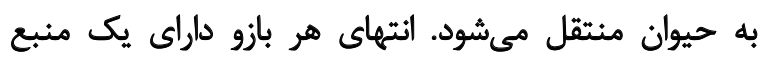

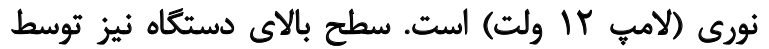

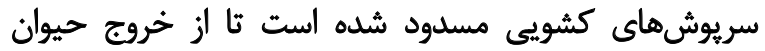

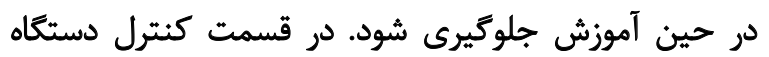

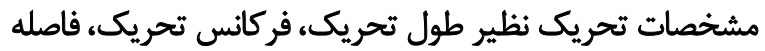

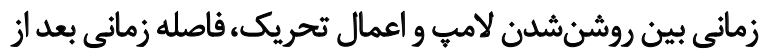

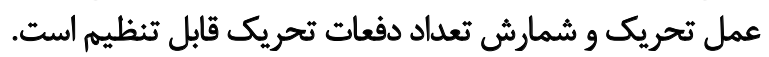

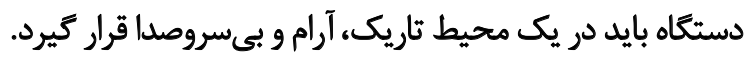
روز اول آموزش به مدت ها دقيقه به حيوان اجازه داده مي آشود

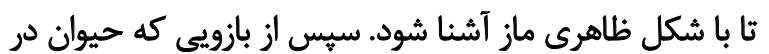

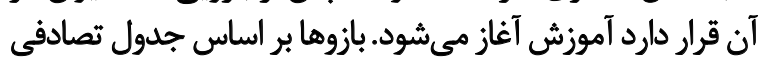

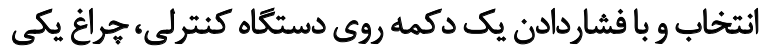

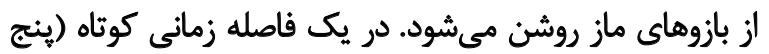

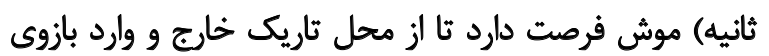
روشن شود؛ در اين صورت ورود موش به بازوى روشن به عاريك عنوان 


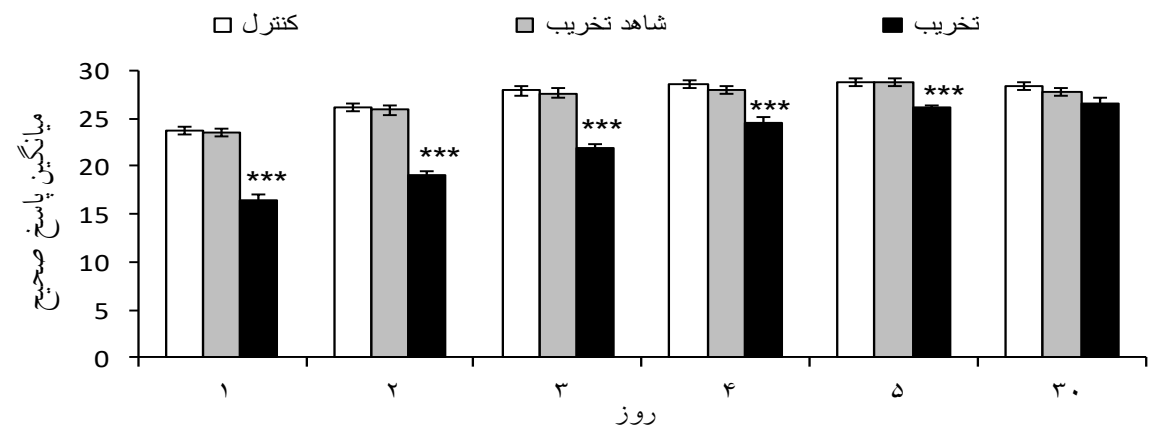

$\sqrt{c}$

تصوير ا. مقايسه ميانكين ياسخ صحيح بين كروه كنترل با كروهماى شاهد تخريب و تخريب در روزهاى اول تا بنجمم و روز سىام با روز بنجم آموزش در موشهاى

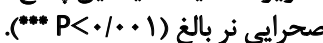
دادهها به صورت ميانكين بانحراف معيار نشان داده شدهائد.

ياسخ صحيح در روزهاى اول، دوم، سوم و جهارم آموزش است.

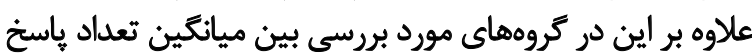

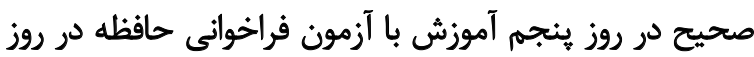
سىام اختلاف معنى دارى مشاهده نشد (تصوير شماره ب). بحث

با توجه به اينكه اثرات ممانتين وويتامين D بلهتنهايى در درمان

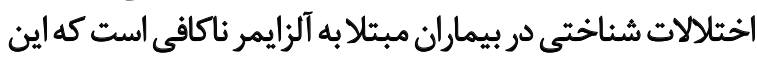

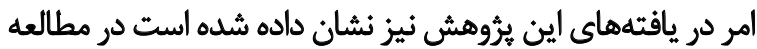

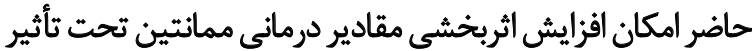

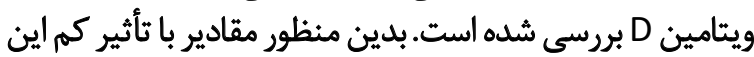

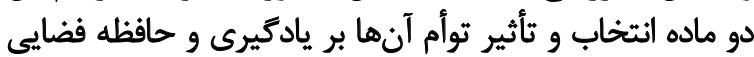

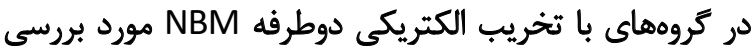

فراخوانى حافظه روز سىام در هركّروه اختلاف معنى دارى وجود

نداشت (تصوير شماره Y).

در بردسى اثرات ويتامين D، ممانتين و ثأثير توأم آنها بر آنا

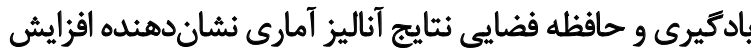

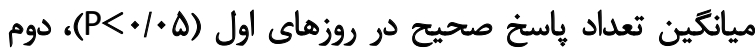

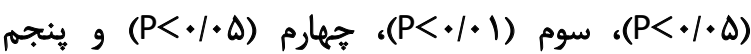

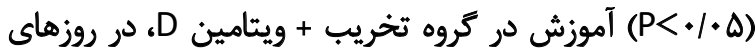

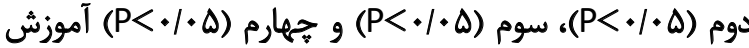

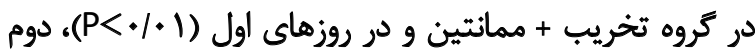

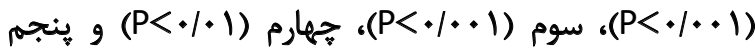

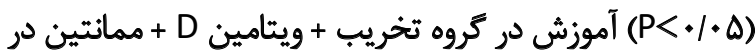

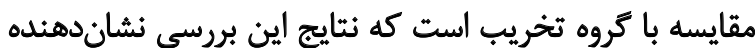

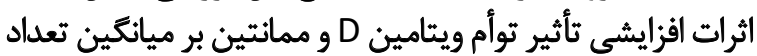

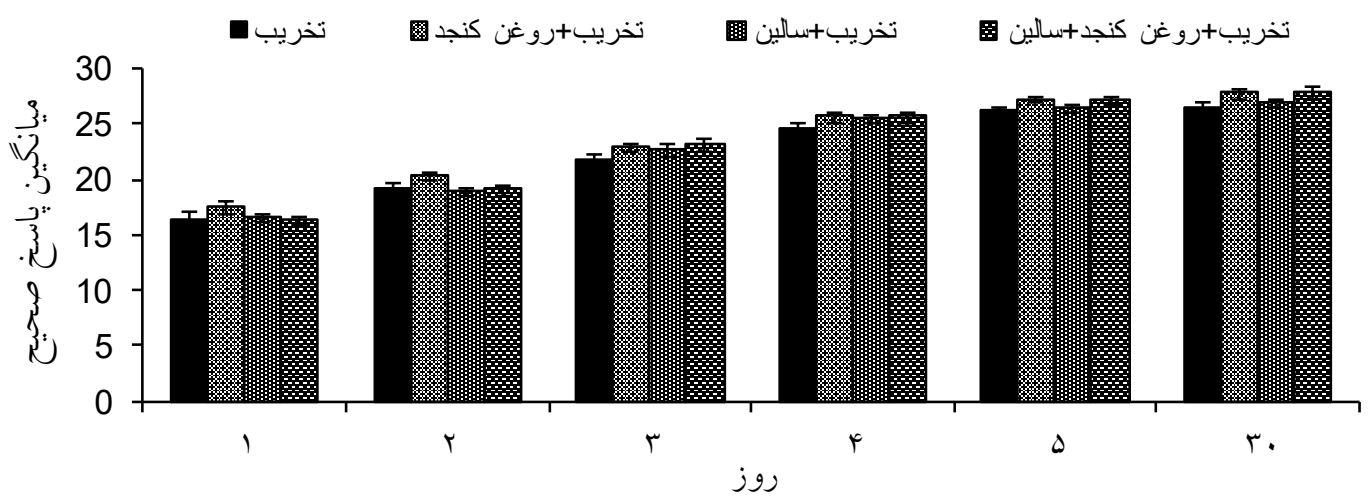

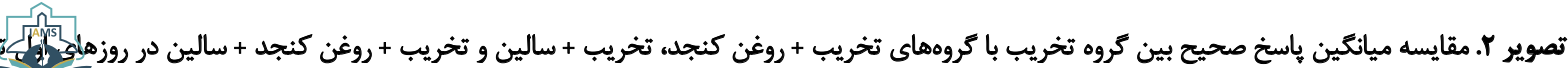

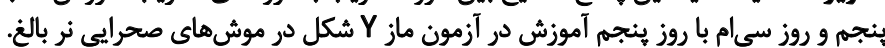
دادهها به صورت ميانكين ذانحراف معيار نشان داده شدهاند. 

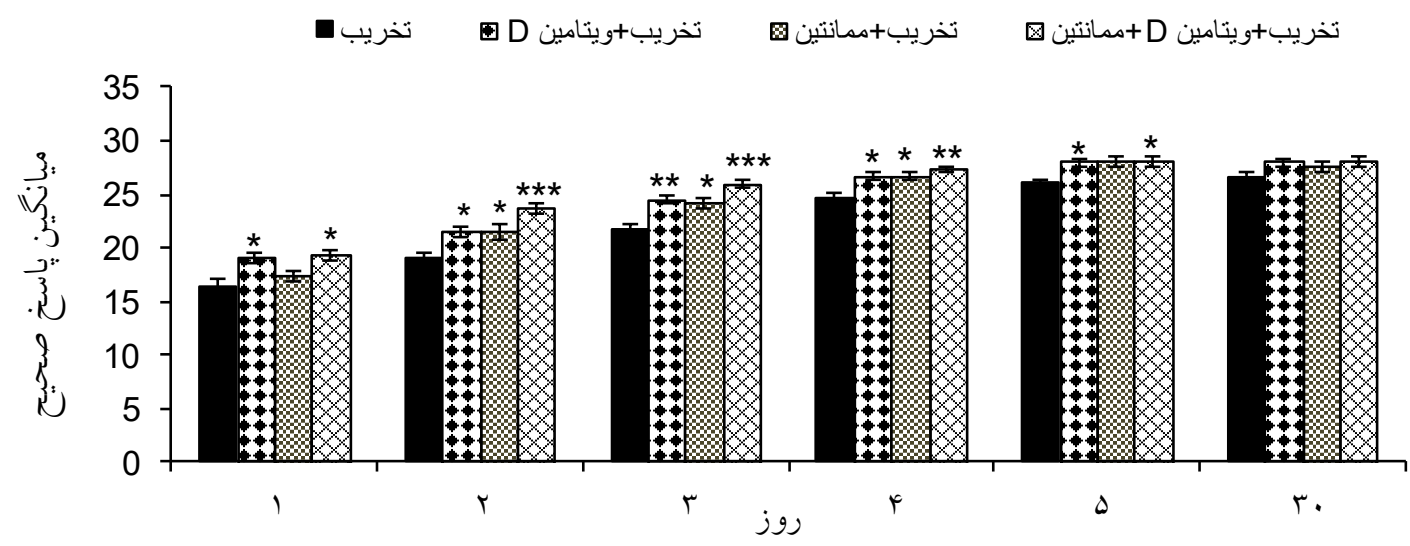

ints

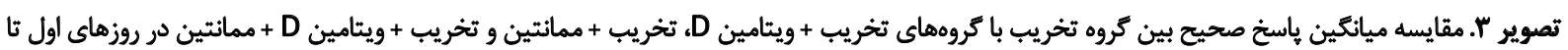

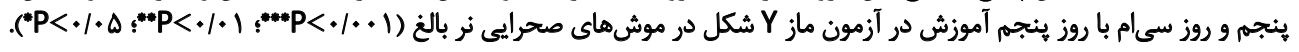

دادهها به صورت ميانكين انانحراف معيار نشان داده شدهائد.

كنجد (حلال ويتامين D)، سالين (حلال ممانتين) و تجويز توأم آنها هيجَّونه تأثيرى بر يادكيرى فضايى ندارد (تصوير شماره T).

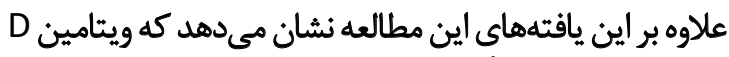

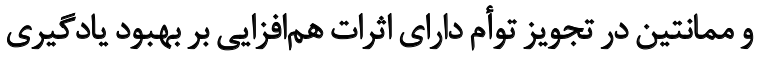

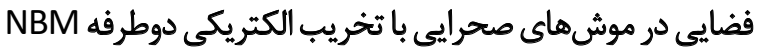

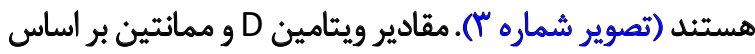

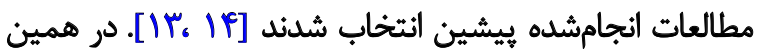

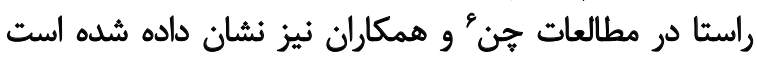

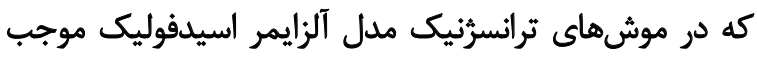

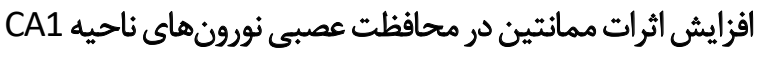

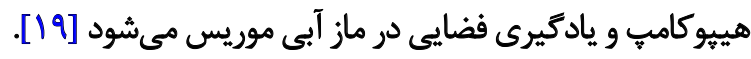

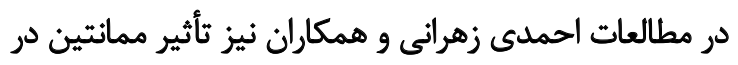

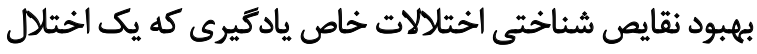

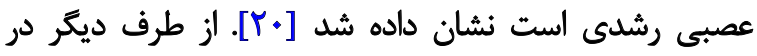

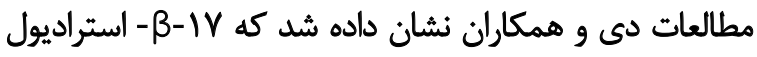

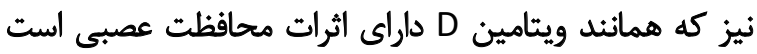

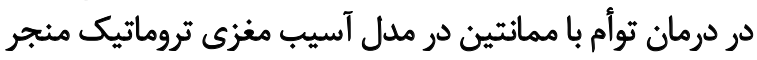

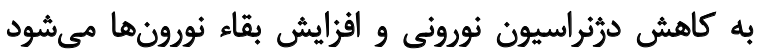

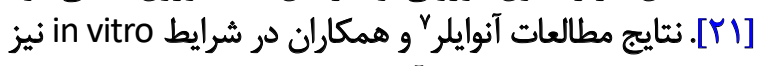

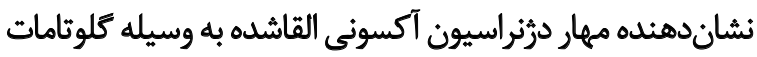

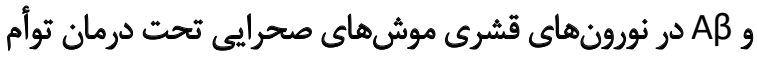

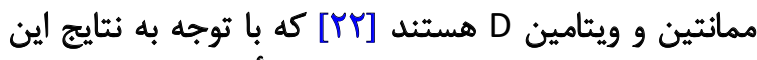

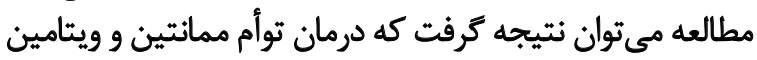
D مؤثرتر از تجويز هريك از اين دو ماده بهتنهاييى است.

6. Chen

7. Annweiler
قرار كرفت. يافتههاى اين مطالعه نشان مى دهند كه درمان توأم

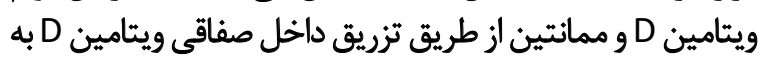

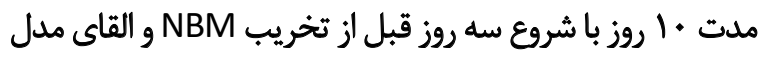

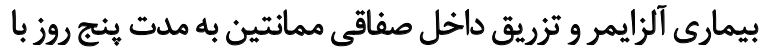

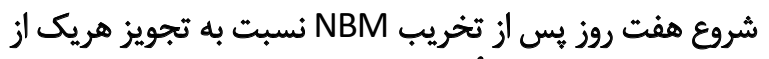

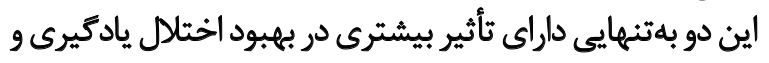

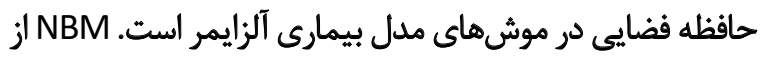

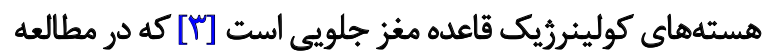

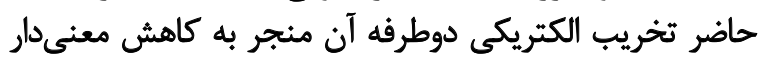
فرايند يادكيرى و حافظه فضايى شد (تصوير شماره ان (). در همين

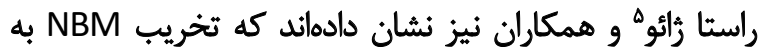

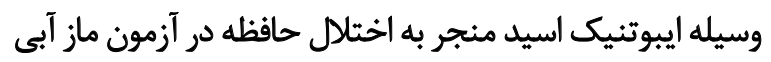

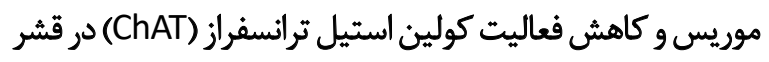

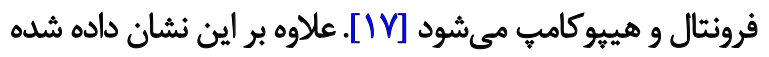

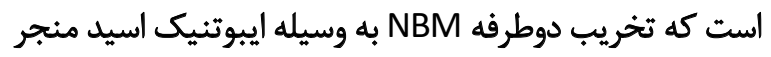
به كاهش سطوح استيل كولين، كولين استراز و كولين استيل

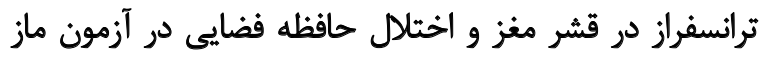

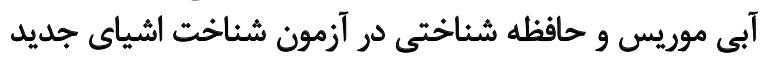

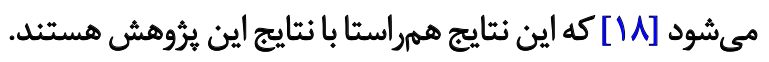
در اين بررسى اختلاف معنى دارى در يادكيرى فضايى

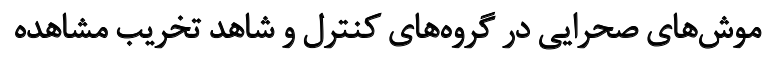

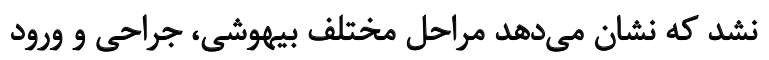

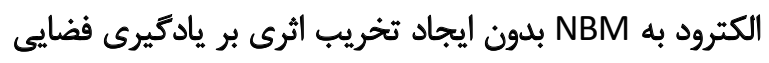

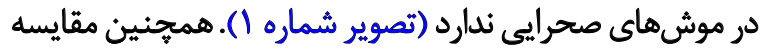

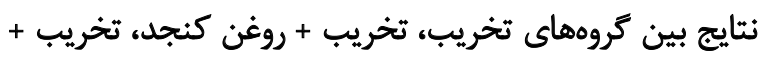
سالين و تخريب + روغن كنجد + سالين نشان داد كه روغن 
فاكوسيتوز AB توسط ماكروفازهاى نوع او ال، بازجذب و كليرانس

A

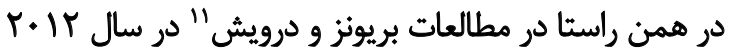

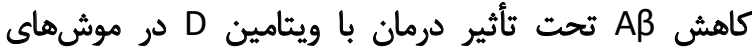

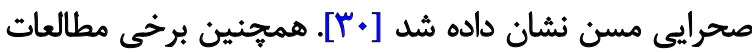

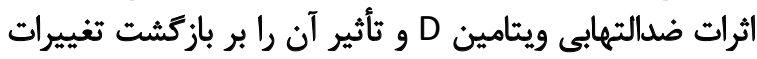

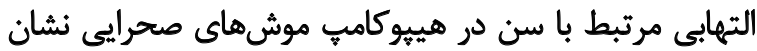

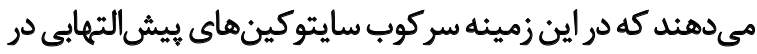

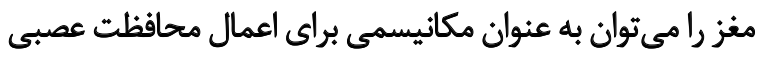

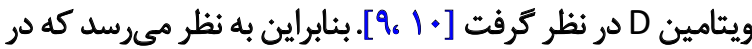

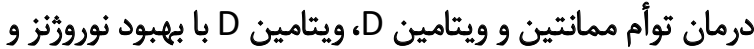

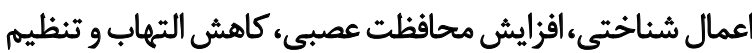

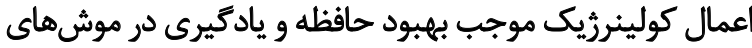
صحرايى مدل آلزايمر و تحت تيمار با ممانتين مى بشود.

\section{نتيجليَّيرى}

در اين مطالعه از تخريب الكتريكى دوطرفه NBM به منه عنوان

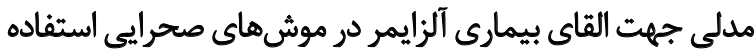

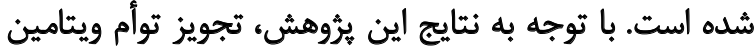

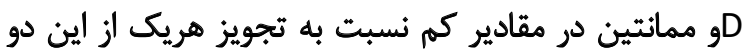

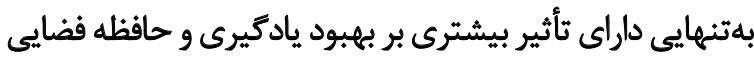
در موشهاى مدل آلزايمر است.

بيشنهاد مىشود كه براي مطالعات بيشتر در اين زمينه اثرات

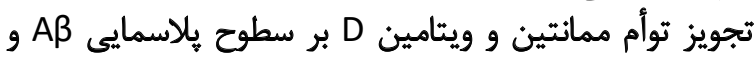

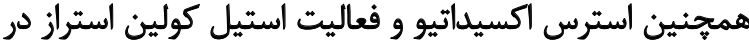

بافت مغزى موشهاى صحرايي مدل آلزايمر بررسى شود.

مالاحظات اخلاقى

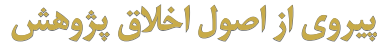

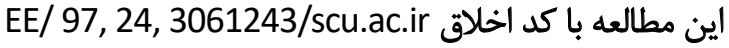
توسط كميته اخلاق يُؤهشى دانشكاه شهيد جمران اهوان اهواز به

تصويب رسيده است.

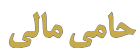

ايـن مقالـهـ از حمايـت مالـى دانشـعاه شـهـيد جمــران اهـواز

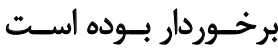

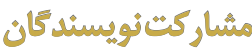

تمامى نويسندكان در نكارش اين مقاله به يك اندازه مشاركت

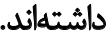

11. Darwish \& Briones
مكانيسم برهمكنش ممانتين و ويتامين D در مدل بيمارى

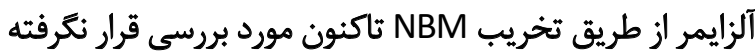

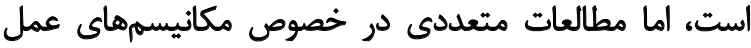

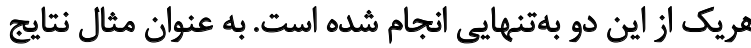

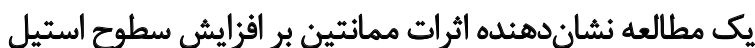

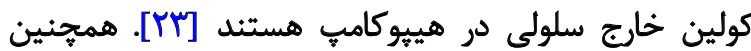

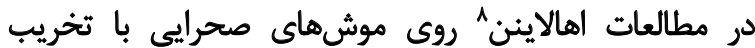
فيمبريافورنيكس افزايش ناجيز سطوح استيل كولين نئوكوري مورتكس

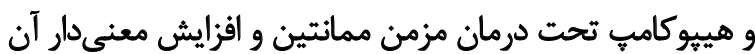

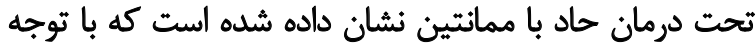

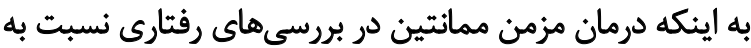

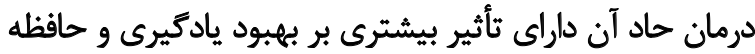

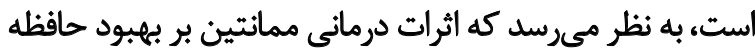

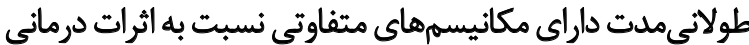

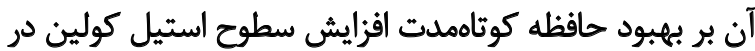

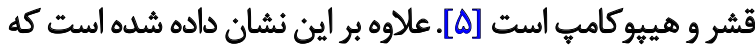

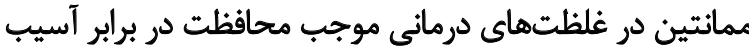

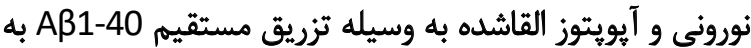

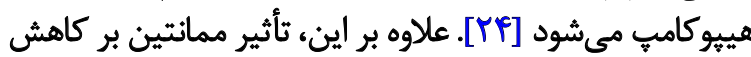

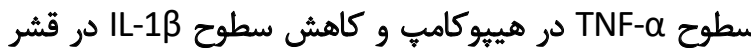

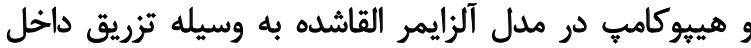

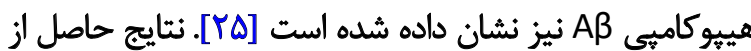

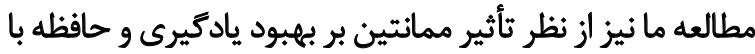
موارد بيش كفته همخوانى داشت.

ازئرف ديكر بهبود نورورثز و اعمال شناختى در موشهاي

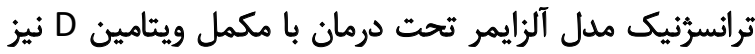

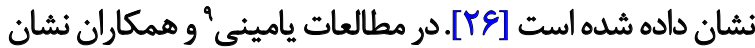

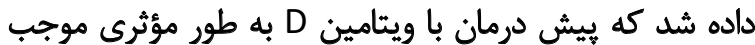

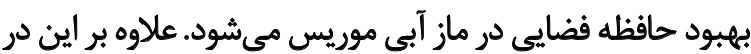

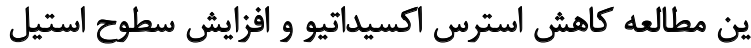

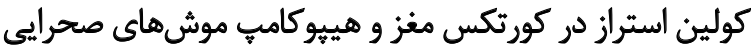

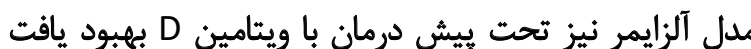

[rV]

Dر بررسىهاى ييوش" و همكاران نيز مكمل ويتامين

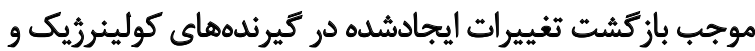

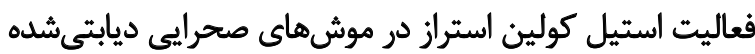

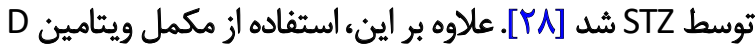

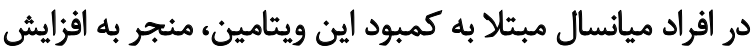

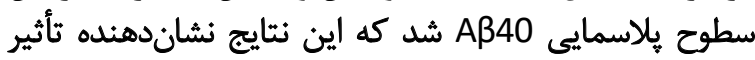

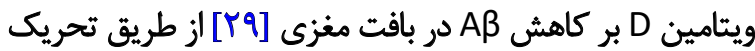


نويسندكًان اعلام مىدارند كه هيجَّونه تضاد منافعى در

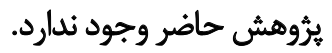

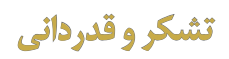

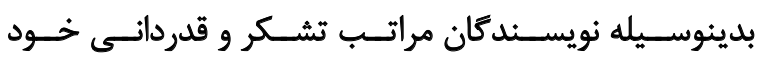

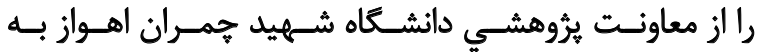

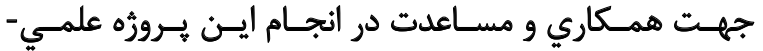

يرؤوهشـي اعـلام مسى دارئسد. 


\section{References}

[1] Agatonovic-Kustrin S, Kettle C, Morton DW. A molecular approach in drug development for Alzheimer's disease. Biomed Pharmacother 2018; 106:553-65. [DOI:10.1016/j.biopha.2018.06.147] [PMID]

[2] Zhao LX, Ge YH, Xiong CH, Tang L, Yan YH, Law PY, et al. M1 muscarinic receptor facilitates cognitive function by interplay with AMPA receptor GluA1 subunit. FASEB J. 2018; 32(8):4247-57. [DOI:10.1096/ fj.201800029R] [PMID]

[3] Nyakas C, Granic I, Halmy LG, Banerjee P, Luiten PG. The basal forebrain cholinergic system in aging and dementia. Rescuing cholinergic neurons from neurotoxic amyloid- $\beta 42$ with memantine. Behav Brain Res. 2011; 221(2):594-603. [DOI:10.1016/j.bbr.2010.05.033] [PMID]

[4] Shearman E, Rossi S, Szasz B, Juranyi Z, Fallon S, Pomara N, et al. Changes in cerebral neurotransmitters and metabolites induced by acute donepezil and memantine administrations: A microdialysis study. Brain Res Bull. 2006; 69(2):204-13. [DOI:10.1016/j.brainresbull.2005.12.001] [PMID]

[5] Ihalainen J, Sarajärvi T, Rasmusson D, Kemppainen S, Keski-Rahkonen $\mathrm{P}$, Lehtonen $\mathrm{M}$, et al. Effects of memantine and donepezil on cortical and hippocampal acetylcholine levels and object recognition memory in rats. Neuropharmacology. 2011; 61(5-6):891-99. [DOI:10.1016/j.neuropharm.2011.06.008] [PMID]

[6] Nagakura A, Shitaka Y, Yarimizu J, Matsuoka N. Characterization of cognitive deficits in a transgenic mouse model of Alzheimer's disease and effects of donepezil and memantine. Eur J Pharmacol. 2013; 703(1-3):5361. [DOI:10.1016/j.ejphar.2012.12.023] [PMID]

[7] Wang YC, Sanchez-Mendoza EH, Doeppner TR, Hermann DM. Post-acute delivery of memantine promotes post-ischemic neurological recovery, peri-infarct tissue remodeling, and contralesional brain plasticity. J Cereb Blood Flow Metab. 2017; 37(3):980-93. [DOI:10.1177/0271678X16648971] [PMID] [PMCID]

[8] Taghizadeh M, Djazayery A, Salami M, Eshraghian MR, Zavareh SA. Vitamin-D-free regimen intensifies the spatial learning deficit in Alzheimer's disease. Int J Neurosci. 2011; 121(1):16-24. [DOI:10.3109/00207454.20 10.523132] [PMID]

[9] Moore ME, Piazza A, McCartney Y, Lynch MA. Evidence that vitamin D3 reverses age-related inflammatory changes in the rat hippocampus. Biochem Soc Trans. 2005; 33(Pt 4):573-77. [DOI:10.1042/BST0330573] [PMID]

[10] Banerjee A, Khemka VK, Ganguly A, Roy D, Ganguly U, Chakrabarti S. Vitamin D and Alzheimer's Disease: Neurocognition to Therapeutics. Int J Alzheimers Dis. 2015; 2015:192747. [DOI:10.1155/2015/192747] [PMID] [PMCID]

[11] Masoumi A, Goldenson B, Ghirmai S, Avagyan H, Zaghi J, Abel K, et al. 1alpha,25-dihydroxyvitamin D3 interacts with curcuminoids to stimulate amyloid-beta clearance by macrophages of Alzheimer's disease patients. J Alzheimers Dis. 2009; 17(3):703-17. [DOI:10.3233/JAD-20091080] [PMID]

[12] Gezen-Ak D, Dursun E, Yilmazer S. The Effect of Vitamin D Treatment On Nerve Growth Factor (NGF) Release From Hippocampal Neurons. Noro Psikiyatr Ars. 2014; 51(2):157-62. [DOI:10.4274/npa.y7076] [PMID] [PMCID]

[13] Mashayekhi F, salehi Z, Mirzajani E. [Effects of vitamin D on myelin basic protein expression in corpus callosum of mouse model of experimental autoimmune encephalomyelitis induced by Cuprizone (Persian)]. Journal of Cell \& Tissue. 2018; 8(4):314-21.
[14] Zamani N, Moazedi AA, Afarinesh Khaki MR, Pourmehdi Boroujeni M. [Effect of memantine on spatial learning andmemory in electrical leisions model of nucleus basalis magnocellularis: Animal model of Alzheimer's disease (Persian)]. J Gorgan Univ Med Sci 2018; 20(1):43-50.

[15] Ashkavandi S, Moazedi AA, Semnanian S, Eshagh-Harooni H, Mohammadi T, Pourmehdi-Boroujeni $\mathrm{M}$. The effect of methylprednisolone on spatial learning and memory in adult male rats using an experimental model of Alzheimer's disease. Feyz, J Kashan Univ of Med Sci 2015; 19(2):102-10.

[16] Hoveida R, Moazedi AA, Rasekh AR. [The effect of intra-Hippocampal CA1 area injection of Estradiol Benzoate on the spatial learning and memory in adult male rats (Persian)]. Modares J Med Sci. 2005; 7(2):115-24.

[17] Zhao Z, Zhao S, Xu N, Yu C, Guan S, Liu X, et al. Lovastatin improves neurological outcome after nucleus basalis magnocellularis lesion in rats. Neuroscience. 2010; 167(3):954-63. [DOI:10.1016/j.neuroscience.2010.02.054] [PMID]

[18] Hou XQ, Zhang L, Yang C, Rong CP, He WQ, Zhang CX, et al. Alleviating effects of Bushen-Yizhi formula on ibotenic acid-induced cholinergic impairments in rat. Rejuvenation Res. 2015; 18(2):111-27. [DOI:10.1089/ rej.2014.1603] [PMID] [PMCID]

[19] Chen TF, Huang RF, Lin SE, Lu JF, Tang MC, Chiu MJ. Folic Acid potentiates the effect of memantine on spatial learning and neuronal protection in an Alzheimer's disease transgenic model. J Alzheimers Dis. 2010; 20(2):607-15. [DOI:10.3233/JAD-2010-1396] [PMID]

[20] Ahmadi Zahrani E, Nokani M, Keypour M, Alaghemand A. [Effectiveness of memantine in improvement of cognitive deficits in specific learning disorder (Persian)]. Arak Med Univ J. 2016; 19(114):1-11.

[21] Day NL, Carle MS, Floyd CL. Post-injury administration of a combination of memantine and $17 \beta$-estradiol is protective in a rat model of traumatic brain injury. Neurochem Int. 2017; 111:57-68. [DOI:10.1016/j. neuint.2017.04.018] [PMID]

[22] Annweiler C, Brugg B, Peyrin JM, Bartha R, Beauchet O. Combination of memantine and vitamin $D$ prevents axon degeneration induced by amyloid-beta and glutamate. Neurobiol Aging. 2014; 35(2):331-35. [DOI:10.1016/j.neurobiolaging.2013.07.029] [PMID]

[23] Yamaguchi Y, Takeda K, Hino M. Combination effects of ZSET1446/ ST101 with memantine on cognitive function and extracellular acetylcholine in the hippocampus. J Pharmacol Sci. 2013; 123(4):347-55. [DOI:10.1254/jphs.13042FP] [PMID]

[24] Miguel-Hidalgo JJ, Alvarez XA, Cacabelos R, Quack G. Neuroprotection by memantine against neurodegeneration induced by betaamyloid (1-40). Brain Res. 2002; 958(1):210-21. [DOI:10.1016/S00068993(02)03731-9]

[25] Budni J, Feijó DP, Batista-Silva H, Garcez ML, Mina F, Belletini-Santos T, et al. Lithium and memantine improve spatial memory impairment and neuroinflammation induced by $\beta$-amyloid 1-42 oligomers in rats. Neurobiol Learn Mem. 2017; 141:84-92. [DOI:10.1016/j.nlm.2017.03.017] [PMID]

[26] Morello M, Landel V, Lacassagne E, Baranger K, Annweiler C, Féron $F$, et al. Vitamin D Improves Neurogenesis and Cognition in a Mouse Model of Alzheimer's Disease. Mol Neurobiol. 2018; 55(8):6463-79. [DOI:10.1007/s12035-017-0839-1] [PMID] [PMCID]

[27] Yamini P, Ray RS, Chopra K. Vitamin D3 attenuates cognitive deficits and neuroinflammatory responses in ICV-STZ induced sporadic Alzheimer's disease. Inflammopharmacology. 2018; 26(1):39-55. [DOI:10.1007/ s10787-017-0372-x] [PMID] 
[28] Peeyush KT, Savitha B, Sherin A, Anju TR, Jes P, Paulose CS. Cholinergic, dopaminergic and insulin receptors gene expression in the cerebellum of streptozotocin-induced diabetic rats: functional regulation with Vitamin D3 supplementation. Pharmacol Biochem Behav. 2010; 95(2):216-22. [DOI:10.1016/j.pbb.2010.01.008] [PMID]

[29] Miller BJ, Whisner CM, Johnston CS. Vitamin D Supplementation Appears to Increase Plasma $A \beta 40$ in Vitamin D Insufficient Older Adults: $A$ Pilot Randomized Controlled Trial. J Alzheimers Dis. 2016; 52(3):843-47. [DOI:10.3233/JAD-150901] [PMID]

[30] Briones TL, Darwish H. Vitamin D mitigates age-related cognitive decline through the modulation of pro-inflammatory state and decrease in amyloid burden. J Neuroinflammation. 2012; 9:244. [DOI:10.1186/17422094-9-244] [PMID] [PMCID] 